\title{
9 \\ On Automatic Calibration of the SWMM Model
}

\author{
Van-Thanh-Van Nguyen, Hamed Javaheri and Shie-Yui Liong
}

Conceptual urban runoff (CUR) models, such as the U.S. Environmental Protection Agency Storm Water Management Model (Huber and Dickinson, 1988), or SWMM, are commonly used for planning and design of urban drainage systems. These models require usually a large number of variables and parameters in order to describe adequately the complex relationships between rainfall, runoff and watershed characteristics. This requirement has frequently become a barrier to the use of these models because of the difficulties involved in the estimation of all the model parameters. More specifically, the successful application of conceptual runoff models depends on how accurate the model is calibrated. However, the calibration of these models has been recognized as a complex and difficult task because of the presence of multiple optimal solutions encountered in the calibration process (Duan et al., 1992; Cooper et al., 1997). Such problem may be caused either by the limitations inherent in the calibration and verification data used, or by the typical nonlinear structure of the models.

In practice, the most commonly used calibration procedure for conceptual runoff models is the trial-and-error approach. This method, however, is inefficient and time consuming, especially when the number of calibrating parameters is large, and could yield inaccurate results. Hence, there is a great need

Nguyen, V., H. Javaheri and S. Liong. 2000. "On Automatic Calibration of the SWMM Model." Journal of Water Management Modeling R206-09. doi: 10.14796/JWMM.R206-09.

(C) CHI 2000 www.chijournal.org ISSN: 2292-6062 (Formerly in Applied Modeling of Urban Water Systems. ISBN: 0-9683681-3-1) 
for a robust and reliable automatic calibration procedure in order to assist in the identification of the best set of optimal parameters for a CUR model. Calibration procedures based on local optimization techniques have been found useful for cases where the response surface is relatively smooth and the optimal solution is unique (Johnston and Pilgrim, 1976; Gan and Bifu, 1966). Calibration procedures based on global optimization methods have been shown to be more robust and more reliable since they can locate the global optimal solution of highly irregular response surfaces (Duan et al., 1992; Cooper et al., 1997). Further, while there have been several attempts to develop automatic calibration procedures for general conceptual rainfall-runoff models (Gupta and Sorooshian, 1985; Wang, 1991; Duan et al., 1992; Gan and Biftu, 1996; Franchini, 1996), there were few studies dealing with the automatic calibration of urban runoff models (see, e.g. Liong et al., 1991). Therefore, appropriate techniques should be developed for solving problems associated with the calibration of CUR models.

In view of the above problems, the main objective of the present study is to propose an automatic calibration scheme for CUR models using an appropriate optimization technique. Two optimization methods were selected: the Downhill Simplex (DHS) method (Nelder and Mead, 1965), and the Shuffled Complex Evolution (SCE) technique (Duan et al., 1992). The DHS is chosen because it is one of the most popular procedures for calibrating hydrologic conceptual models. If available information regarding the mathematical properties of the objective function indicates that there is only one optimal value for a given feasible parameter range, then the use of a local optimization technique such as the DHS could be appropriate. Such information, however, is often not available for most practical problems. In such cases, the calibration problem should be treated as a global optimization one. As shown by some recent studies (Duan et al., 1992; Cooper et al., 1997), global optimization methods such as the SCE are capable of handling the existence of multi-local optima in the calibration of conceptual hydrologic models.

The proposed automatic calibration procedures were applied to the SWMM model. Two different scenarios were considered using "error-free" synthetic data, and using observed data available on the Upper Bukit Timah catchment in Singapore. Results of this study have indicated that, for the SWMM model, there are many local optima within a given feasible parameter range, and hence the use of the DHS local optimization technique would not be appropriate. In such cases, the calibration problem should be treated as a global optimization one. More specifically, it has been shown that the proposed SCE-based calibration scheme was able to provide consistent parameter estimates for the SWMM model. In summary, the proposed automatic calibration procedure is more efficient and more accurate than the trial-and-error and local optimization-based calibration methods, and it is capable of providing a unique set of optimal parameters. 


\subsection{Methodology}

SWMM is one of the most widely accepted models for planning, design and management of urban drainage systems. This comprehensive conceptual urban runoff model (CUR) is capable of water quality and quantity simulations, and for both storm or combined sewer systems. All features of the hydrologic or qualitative cycles are simulated, in order to provide an accurate prediction. A detailed description of SWMM can be found in the literature (Huber and Dickinson, 1992; Nix, 1994; Viessman and Lewis, 1996). In general, SWMM consists of two fundamental groups, the computational blocks which include the Runoff, Transport, Extended Transport (Extran) and Storage/Treatment Blocks, and the service blocks which include the Executive, Combine, Statistics, Rain, Temp and Graph blocks. The present study focuses on the capability of SWMM in water quantity simulation and, therefore, only that portion of the Runoff block was considered.

The Runoff block may be run for short duration storms (single events), or for a longer time period including several storms (continuous simulation). The input data requirements for the Runoff Block are quite substantial and detailed, and include hydraulic and geometric properties of the drainage network as well as rainfall and watershed physiographic characteristics. The watershed being considered is divided into several sub-catchments. These sub-catchments are mathematically idealized as spatially lumped, nonlinear reservoirs, and their flows are routed through the channel to which they attached (Huber and Dickinson, 1992). With such a large data requirement, SWMM's application is often limited to small urban watersheds with well-known characteristics.

As mentioned above, the watershed considered in this study is the Upper Bukit Timah (UBT) catchment located in Singapore. This catchment covers an area of $6.11 \mathrm{~km}^{2}$, of which approximately $35 \%$ is developed. A forest reserve, an essentially pervious (90-100\%) and steep sloped (6-10\%) rough surfaced area, exists at the catchment uplands. The remaining area is relatively flat (roughly $4 \%$ slope) and consists of low-rise residential areas with some commercial and business buildings having approximately $16-30 \%$ impervious areas. The UBT watershed is divided into 19 sub-catchments. The sub-catchment and gutter characteristics have been described in more detail in a study by Javaheri (1999).

Table 9.1 describes the eight parameters that were considered in the calibration of SWMM using the data available on the UBT catchment. These parameters were selected because they were found to be sensitive to the model output, based on the sensitivity analysis results obtained in several previous studies (Ibrahim and Liong, 1992, 1993; Liong and Ibrahim, 1994; and Liong et al., 1995) for the same watershed. Further, as required by the search process of the calibration algorithm, a range of possible values for each parameter must be defined. Hence, it was considered important to determine the allowable range of 
Table 9.1 Calibration parameters.

\begin{tabular}{|c|c|c|}
\hline Parameter & Corresponding Variable & Initial Variable Estimates \\
\hline $\mathrm{X} 1$ & $\begin{array}{l}\text { Manning's roughness of pervious } \\
\text { area }\end{array}$ & 0.30 \\
\hline $\mathrm{X} 2$ & $\begin{array}{l}\text { Depression storage of pervious } \\
\text { area }\end{array}$ & $7.5 \mathrm{~mm}$ \\
\hline $\mathrm{X} 3$ & Initial infiltration rate (Horton's) & $45 \mathrm{~mm} / \mathrm{hr}-80 \mathrm{~mm} / \mathrm{hr}$ \\
\hline $\mathrm{X} 4$ & $\begin{array}{l}\text { Ultimate infiltration rate } \\
\text { (Horton's) }\end{array}$ & $2.5 \mathrm{~mm} / \mathrm{hr}-3.5 \mathrm{~mm} / \mathrm{hr}$ \\
\hline $\mathrm{X} 5$ & Infiltration decay rate (Horton's) & $0.00005 / \mathrm{sec}-0.0011 / \mathrm{sec}$ \\
\hline $\mathrm{X} 6$ & Sub-catchment width & $\begin{array}{l}\text { Varies with sub-catchment } \\
(92 \mathrm{~m}-2132 \mathrm{~m})\end{array}$ \\
\hline $\mathrm{X} 7$ & Percent impervious area & $\begin{array}{l}\text { Varies with sub-catchment } \\
(0.03-0.376)\end{array}$ \\
\hline $\mathrm{X} 8$ & Sub-catchment slope & $\begin{array}{l}\text { Varies with sub-catchment } \\
(0.02-0.10)\end{array}$ \\
\hline
\end{tabular}

parameter values such that the estimated parameters should be realistic in value. Therefore, based on various recommendations in the literature (e.g. Huber and Dickinson, 1992; WP-Software, 1993), and on the basis of previous studies by Ibrahim and Liong (1992), and Liong et al. (1995) for the UBT watershed, a realistic and physically meaningful range of values for each parameter and corresponding variable was defined as shown in Table 9.2.

Table 9.2 Upper and lower bounds of calibration parameters and variables.

\begin{tabular}{lll}
\hline Parameter & \multicolumn{1}{c}{ Range } & Range of Allowable Values of Corresponding Variable \\
\hline $\mathrm{X} 1$ & $0.1-1.7$ & $0.03-0.51$ \\
$\mathrm{X} 2$ & $0.2-1.4$ & $1.5 \mathrm{~mm}-10.5 \mathrm{~mm}$ \\
$\mathrm{X} 3$ & $0.15-2.0$ & $6.75 \mathrm{~mm} / \mathrm{hr}-160 \mathrm{~mm} / \mathrm{hr}$ \\
$\mathrm{X} 4$ & $0.1-2.0$ & $0.0 \mathrm{~mm} / \mathrm{hr}-11.3 \mathrm{~mm} / \mathrm{hr}$ \\
$\mathrm{X} 5$ & $0.7-2.0$ & $0.000035 / \mathrm{sec}-0.0022 / \mathrm{sec}$ \\
$\mathrm{X} 6$ & $0.5-1.5$ & $\pm 50 \%$ of the initial value \\
$\mathrm{X} 7$ & $0.5-1.5$ & $\pm 50 \%$ of the initial value \\
$\mathrm{X} 8$ & $0.5-1.5$ & $\pm 50 \%$ of the initial value
\end{tabular}

Table 9.3 provides a summary of the characteristics of the twelve rainfall events considered. These different storms, six multiple burst (MB) and six single burst (SB) storms, were chosen to represent the wide range of rainfall characteristics in Singapore.

The calibration of SWMM can be considered as an optimization problem whose objective is to determine the optimal values of the model parameters in order to provide the best fit between observed and estimated runoffs. The fit between the measured and predicted values is assessed using an objective 
Table 9.3 Storm characteristics.

\begin{tabular}{ccccc} 
Storm & Time Started & $\begin{array}{c}\text { Duration } \\
(\mathrm{hrs})\end{array}$ & $\begin{array}{c}\text { Peak } \\
\left(\mathrm{m}^{3} / \mathrm{s}\right)\end{array}$ & Date \\
\hline MB1 & $16: 00$ & 28.25 & 21.5 & $2 / 3 / 84$ \\
MB2 & $00: 00$ & 29.75 & 31.0 & $2 / 12 / 78$ \\
MB3 & $13: 00$ & 23.25 & 0.8 & $25 / 1 / 87$ \\
MB4 & $17: 00$ & 14.25 & 7.9 & $1 / 3 / 85$ \\
MB5 & $16: 00$ & 19.75 & 3.9 & $7 / 2 / 84$ \\
MB6 & $11: 00$ & 44.00 & 14.2 & $8 / 1 / 86$ \\
SB1 & $11: 15$ & 7.00 & 18.3 & $1 / 6 / 79$ \\
SB2 & $03: 00$ & 16.75 & 21.4 & $7 / 10 / 86$ \\
SB3 & $15: 30$ & 7.50 & 19.8 & $28 / 2 / 80$ \\
SB4 & $02: 30$ & 9.75 & 26.0 & $15 / 2 / 88$ \\
SB5 & $00: 00$ & 20.25 & 18.6 & $16 / 1 / 88$ \\
SB6 & $11: 00$ & 12.75 & 9.8 & $22 / 9 / 87$ \\
\hline
\end{tabular}

function. Based on the goodness of fit in each iteration, the optimization algorithm decides the direction in which the search should be continued, and the parameter values that can be used for the next iteration. These parameter values are repeatedly refined until the difference between the simulated and measured hydrographs is within an acceptable user-defined tolerance. The iterative process of parameter estimation requires a calibration algorithm that incorporates an objective function value to each iteration. When the stopping condition is fulfilled, the best set of parameters found in the search is assumed as optimal.

Initially, the local optimization DHS technique (Nelder and Mead, 1965) was used to calibrate SWMM. The Nash coefficient was chosen as the objective function since it could provide the best estimates of conceptual model parameters as compared to other goodness-of-fit criteria (Cooper et al., 1997). This coefficient is defined as follows:

$$
N A S H=1-\frac{\sum\left(Q_{s}-Q_{m}\right)^{2}}{\sum\left(Q_{m}-\bar{Q}_{m}\right)^{2}}
$$

in which $Q_{s}$ is the simulated flow, $Q_{m}$ is the measured flow, and $\overline{Q_{m}}$ is the mean of the measured flows. Hence, a Nash coefficient value of 1.0 represents the perfect fit.

The SWMM and DHS computer codes were combined to form the DHSSWMM calibration procedure. Several iterations were carried out from different points in the feasible region in order to establish the characteristics of the resulting response surface. It is thus possible to ascertain whether the response surface was smooth with only one optimal solution, or whether it was irregular with many local optima. If only one optimal solution exists, the same set of optimal parameters will result, irrespective of the initial starting point. Otherwise, if there 
are many local optima, the DHS may get trapped in a local optimal region and converges to a local optimal solution, instead of a global one. In order to ensure that the DHS can find the best solution, the iteration process is stopped when it has passed through 50 loops with no change in the Nash coefficient value, or when a $10^{-9}$ tolerance level of the Nash coefficient has been achieved.

Similarly, the calibration of SWMM was performed using the global optimization SCE technique (Duan et al., 1992) and using the Nash coefficient as objective function. The SWMM and SCE computer codes were combined to form the SCE-SWMM calibration method. To assess the performance of the SCE-SWMM procedure, as compared to the DHS-SWMM, in the search for the global optimal solution, different calibration runs from different starting points in the feasible region were carried out for several storms. If only one set of optimal parameters was obtained, independent of the starting location chosen, the resulting optimal solution can be expected as the global optimal one.

\subsection{Results and Discussion}

The DHS-SWMM calibration procedure was applied to the SWMM model considering all eight calibrating parameters and using real rainfall and runoff data. Several simulation runs were carried out using different starting seed values; that is, each run started at a different initial position in the feasible parameter space. In general, the DHS-SWMM procedure can provide a very good fit between observed and simulated flows as indicated, for instance, in Table 9.4 for two storms MB1, and SB2 with Nash coefficient values mostly above 0.80 . However, it can be seen that, for the different seed values selected, different sets of optimal values of the eight parameters were obtained. The presence of different optimal solutions means that the response surface corresponding to the Nash coefficient is irregular and has many local optima. Therefore, to efficiently find the best parameter set out of these many local optima, a global optimization technique is more appropriate.

Further, notice that many of the parameter values as shown in Table 9.4 are converging to their specified limits (the feasible limits suggested in the literature as indicated in Table 9.2). This convergence is not desirable since the optimal values of the parameters should not be equal to the limits imposed initially on them for the search process. Hence, the DHS-SWMM is not quite robust since, depending on the starting location, it may get trapped in the local optimal region and unable to provide the global optimal solution within the realistic range of the parameter values.

The SCE-SWMM procedure was applied to the SWMM model using the real rainfall and runoff data as described above for the DHS-SWMM method. In order to compare the performance of these two calibration methods, the same upper 
Table 9.4 Parameter estimates based on DHS-SWMM procedure for MB1 and SB2 storms using different seeds (numbers in parentheses indicate limiting values).

\begin{tabular}{|c|c|c|c|c|c|c|c|c|c|c|}
\hline Storm & $\begin{array}{l}\text { Seed } \\
\text { Number }\end{array}$ & $\mathrm{X} 1$ & $X 2$ & $\mathrm{X} 3$ & $\mathrm{X} 4$ & $\times 5$ & $\mathrm{X} 6$ & $X 7$ & $\mathrm{X} 8$ & Nash \\
\hline \multirow[t]{3}{*}{ MB1 } & $\# 1$ & 1.053 & $(0.206)$ & 1.325 & 0.364 & 1.152 & $(0.506)$ & (1.499) & $(0.520)$ & 0.89 \\
\hline & $\# 3$ & 1.042 & $(0.216)$ & 0.988 & 0.266 & 0.819 & $(0.506)$ & (1.481) & 0.544 & 0.89 \\
\hline & $\# 4$ & 0.886 & 0.793 & 1.938 & 0.359 & 1.743 & $(0.512)$ & (1.478) & 0.538 & 0.89 \\
\hline \multirow{2}{*}{ SB2 } & $\# 3$ & 0.453 & (1.397) & 0.176 & 0.117 & 1.105 & $(0.504)$ & $(0.501)$ & $(0.505)$ & 0.88 \\
\hline & $\# 4$ & 0.477 & (1.399) & $(0.150)$ & 0.126 & $(0.700)$ & $(0.500)$ & $(0.501)$ & $(0.512)$ & 0.88 \\
\hline
\end{tabular}

Table 9.5 Parameter estimates based on SCE-SWMM procedure for MB1 and SB2 storms using different seeds (numbers in parentheses indicate limiting values).

\begin{tabular}{|c|c|c|c|c|c|c|c|c|c|c|}
\hline Storm & $\begin{array}{c}\text { Seed } \\
\text { Number } \\
\end{array}$ & $\mathrm{X} 1$ & $\mathrm{X} 2$ & $\mathrm{X} 3$ & $\mathrm{X} 4$ & $\times 5$ & $\mathrm{X} 6$ & $\mathrm{X} 7$ & $X 8$ & Nash \\
\hline \multirow[t]{4}{*}{$\mathrm{MBI}$} & $\# 1$ & 1.116 & $(0.202)$ & 1.045 & 0.363 & 0.920 & $(0.504)$ & $(1.500)$ & 0.521 & 0.89 \\
\hline & $\# 2$ & 1.109 & $(0.202)$ & 1.152 & 0.357 & 1.006 & $(0.504)$ & $(1.500)$ & 0.521 & 0.89 \\
\hline & \#3 & 1.151 & 0.216 & 0.930 & 0.411 & 0.861 & 0.511 & 1.450 & 0.508 & 0.89 \\
\hline & $\# 4$ & 1.110 & $(0.203)$ & 1.108 & 0.353 & 0.972 & 0.509 & $(1.499)$ & 0.510 & 0.89 \\
\hline \multirow[t]{4}{*}{ SB2 } & $\# 1$ & 0.474 & $(1.399)$ & $(0.151)$ & $(0.100)$ & 0.719 & $(0.500)$ & $(0.500)$ & $(0.500)$ & 0.88 \\
\hline & $\# 2$ & 0.475 & $(1.397)$ & $(0.151)$ & $(0.100)$ & 0.718 & $(0.500)$ & $(0.501)$ & $(0.500)$ & 0.88 \\
\hline & $\# 3$ & 0.474 & $(1.395)$ & $(0.151)$ & $(0.100)$ & 0.718 & $(0.500)$ & $(0.501)$ & $(0.501)$ & 0.88 \\
\hline & $\# 4$ & 0.474 & (1.397) & $(0.150)$ & $(0.100)$ & 0.718 & $(0.500)$ & $(0.501)$ & $(0.500)$ & 0.88 \\
\hline
\end{tabular}


and lower limits of the eight parameters as given in Table 9.2 were used. The results as shown in Table 9.5 for storms MB1 and SB1 indicated that a unique set of optimal parameter values has been obtained for different seeds (i.e., different starting solutions). As compared with the results obtained by the DHSSWMM method (see Table 9.4), it can be seen that, while both sets of results have some parameter values converge to the imposed limits, in general the nonconverged parameter values of the SCE-SWMM technique remain constant for different seeds. Therefore, the SCE-SWMM procedure is more robust since it is able to find a unique global optimal solution. This conclusion is true even when the parameters do not converge to the limits, as will be shown later using synthetic data.

The above assessment of the SCE-SWMM procedure using real data did not clearly indicate whether the convergence of some parameters to their limiting values and the different optimal solutions obtained for various storms are due to the weakness of the SCE algorithm or due to the random noise inherent in the real data considered. Hence, it is necessary to perform an assessment of the SCESWMM method using "error-free" synthetic data. The synthetic flow series were generated using the real storm data as input to the SWMM model with known parameter values. In such case, the resulting synthetic flow data can be considered as error-free because they were created from known rainfall input data and from a runoff model with known parameters. Further, in this assessment study the initial parameter limits were selected as from 0.001 to 4.0 for parameters X1-X5, and from 0.5 to 1.5 for parameters X6-X8. These widerange constraints were used in order to see if the optimal solution would take on non-realistic parameter values and to see whether any parameter would converge to its specified limits.

For purposes of illustration, Tables 9.6 and 9.7 present the results of the calibration of the SWMM model based on the DHS-SWMM and SCE-SWMM procedures using the synthetic flow series corresponding to the SB2 storm for several different seeds. It can be seen that the SCE-based calibration method is more robust than the DHS because it can obtain a unique global optimal solution regardless of the starting point. The robustness of the proposed SCE-SWMM method was supported as well by the calibration results presented in Table 9.8 for several different storms. Therefore, the results based on synthetic data reconfirms the conclusion stated above, using real rainfall and runoff data, regarding the superior performance of the SCE-SWMM automatic calibration procedure in its ability to find a unique global optimal solution.

Notice further that there is no convergence to the limits occurred for any of the parameters estimated by the SCE-SWMM method as shown in Table 9.8. Therefore, the convergence of the parameters to their limits is not due to the limitation of the SCE algorithm, but could probably be related to the uncertainty associated with the real data. 
Table 9.6 Calibration by DHS-SWMM procedure using synthetic data for SB2 storm for different seeds.

\begin{tabular}{cccccccccc}
\hline Parameter & \multicolumn{10}{c}{ Seed Number } \\
\hline & $\# 1$ & $\# 2$ & $\# 3$ & $\# 4$ & $\# 5$ & $\# 6$ & $\# 7$ & $\# 8$ & \# \\
X1 & 1.115 & 0.670 & 0.660 & 0.987 & 0.697 & 0.393 & 0.966 & 0.499 & 0.732 \\
X2 & 0.446 & 0.355 & 1.899 & 1.367 & 0.376 & 0.461 & 0.302 & 0.805 & 0.384 \\
X3 & 1.270 & 1.468 & 1.060 & 1.045 & 1.319 & 1.300 & 1.265 & 1.295 & 1.291 \\
X4 & 1.550 & 1.649 & 1.024 & 1.262 & 1.524 & 1.245 & 1.458 & 1.437 & 1.476 \\
X5 & 1.619 & 1.950 & 1.644 & 1.924 & 1.419 & 1.079 & 1.210 & 1.572 & 1.305 \\
X6 & 1.380 & 0.827 & 1.947 & 1.484 & 0.875 & 0.501 & 1.961 & 0.729 & 1.099 \\
X7 & 0.682 & 0.719 & 0.705 & 0.700 & 0.695 & 0.722 & 0.671 & 0.716 & 0.687 \\
X8 & 1.390 & 0.945 & 1.517 & 0.885 & 1.614 & 1.888 & 0.851 & 1.487 & 1.400 \\
Nash & 1.0000 & 1.000 & 0.998 & 0.999 & 1.000 & 0.999 & 1.000 & 1.000 & 1.000 \\
\hline
\end{tabular}

Table 9. 7 Calibration by SCE-SWMM procedure using synthetic data for SB2 storm for different seeds.

\begin{tabular}{|c|c|c|c|c|c|c|c|c|c|}
\hline \multirow[t]{2}{*}{ Parameter } & \multicolumn{9}{|c|}{ Seed } \\
\hline & 1811 & 2811 & 3811 & 4811 & 5811 & 6811 & 7811 & 8811 & 9811 \\
\hline $\mathrm{X} 1$ & 0.698 & 0.678 & 0.719 & 0.722 & 0.703 & 0.704 & 0.704 & 0.664 & 1.779 \\
\hline $\mathrm{X} 2$ & 0.493 & 0.719 & 0.600 & 0.545 & 0.765 & 0.676 & 0.676 & 0.710 & 1.580 \\
\hline $\mathrm{X} 3$ & 1.300 & 1.310 & 1.299 & 1.294 & 1.302 & 1.310 & 1.310 & 1.321 & 1.261 \\
\hline$X 4$ & 1.496 & 1.497 & 1.501 & 1.501 & 1.494 & 1.513 & 1.513 & 1.506 & 1.327 \\
\hline$\times 5$ & 1.451 & 1.800 & 1.618 & 1.533 & 1.827 & 1.776 & 1.776 & 1.804 & 2.740 \\
\hline $\mathrm{X} 6$ & 2.088 & 0.929 & 0.993 & 1.040 & 1.191 & 1.089 & 1.089 & 0.953 & 2.671 \\
\hline$x 7$ & 0.696 & 0.704 & 0.699 & 0.696 & 0.704 & 0.704 & 0.704 & 0.706 & 0.692 \\
\hline$x 8$ & 0.312 & 1.385 & 1.344 & 1.296 & 0.904 & 1.014 & 1.014 & 1.199 & 2.768 \\
\hline Nash & 1.000 & 1.000 & 1.000 & 1.000 & 1.000 & 1.000 & 1.000 & 1.000 & 0.999 \\
\hline
\end{tabular}


Table 9.8 Estimated and real values of parameters for different storms using SCE-SWMM method and synthetic data.

\begin{tabular}{|c|c|c|c|c|c|c|c|c|c|c|}
\hline \multicolumn{10}{|c|}{ Estimated Value } & \multirow{2}{*}{$\begin{array}{l}\text { Real } \\
\text { Value }\end{array}$} \\
\hline & MB1 & MB2 & MB3 & MB4 & MB5 & MB6 & SB1 & SB2 & SB3 & \\
\hline $\mathrm{X} 1$ & 0.7040 & 0.7134 & 1.6359 & 0.7194 & 2.2216 & 0.7004 & 0.7071 & 0.6501 & 0.6673 & 0.7 \\
\hline $\mathrm{X} 2$ & 0.5965 & 0.5955 & 1.8639 & 0.5705 & 2.1886 & 0.5979 & 0.7599 & 0.7495 & 0.5474 & 0.6 \\
\hline $\mathrm{X} 3$ & 1.2982 & 1.3015 & 2.1681 & 2.0019 & 2.3171 & 1.2999 & 1.8277 & 1.3413 & 1.6491 & 1.3 \\
\hline $\mathrm{X} 4$ & 1.5004 & 1.4990 & 1.8625 & 1.4942 & 1.8501 & 1.5002 & 1.5332 & 1.5186 & 1.5304 & 1.5 \\
\hline $\mathrm{X} 5$ & 1.5984 & 1.6020 & 2.0967 & 2.2552 & 2.2046 & 1.5997 & 2.7378 & 1.9334 & 2.0282 & 1.6 \\
\hline $\mathrm{X} 6$ & 1.0649 & 1.1707 & 1.1154 & 1.0516 & 1.0785 & 1.0564 & 1.1800 & 1.0614 & 0.9776 & 1.2 \\
\hline $\mathrm{X} 7$ & 0.6994 & 0.6982 & 0.6997 & 0.7000 & 0.6998 & 0.6997 & 0.7040 & 0.7103 & 0.7050 & 0.7 \\
\hline $\mathrm{X} 8$ & 1.1555 & 0.9844 & 1.0405 & 1.1869 & 1.1135 & 1.1647 & 0.9088 & 0.8874 & 1.1827 & 0.9 \\
\hline Nash & 1.0000 & 1.0000 & 1.0000 & 1.0000 & 1.0000 & 1.0000 & 1.0000 & 1.0000 & 1.0000 & \\
\hline
\end{tabular}




\subsection{Conclusions}

Two automatic calibration procedures (DHS-SWMM and SCE-SWMM) have been proposed for calibrating the SWMM model. Results of the comparison of these two procedures using synthetic and real data have indicated the superior performance of the SCE-based method in terms of accuracy and robustness of the estimated model parameters. As the response surface of the SWMM was highly irregular, the local optimization DHS method was found trapped in a localized optimal region and converged to a local optimal solution. The global optimization SCE method, however, was able to provide more accurate parameter estimates, and consequently, a better fit between estimated and observed hydrographs for the SWMM model.

Further, it should be noted that the calibration of CUR models can be more efficiently achieved if a minimum number of significant parameters could be identified since simpler search procedure is required for finding the global optimal solution when a smaller number of parameters are involved. Sensitivity analysis, therefore, should be performed to be able to identify an optimal set of significant parameters for calibration. For the application of the SWMM to the Upper Bukit Timah watershed, it has been found that the automatic calibration based on two parameters X6 (the sub-catchment width) and X7 (the percent impervious area) was able to provide results comparable to those given by the calibration using all eight parameters (Javaheri, 1999).

\section{References}

Cooper, V. A., Nguyen, V. T. V., and Nicell, J. A. (1997). "Evaluation of global optimization methods for conceptual rainfall runoff model calibration." Water Science and Technology, 36(5), 53-60.

Duan, Q., Sorooshian, S., and Gupta, V. (1992). "Effective and efficient global optimization for conceptual rainfall-runoff models." Water Resources Research, 28(4), 1015-1031.

Franchini, M. (1996). "Use of a genetic algorithm combined with a local search method for the automatic calibration of conceptual rainfall-runoff models." Hydrological Sciences Journal, 41(1), 21-39.

Gan, T. Y., and Biftu, G. F. (1996). "Automatic calibration of conceptual rainfall-runoff models: optimization algorithms, catchment conditions, and model structure." Water Resources Research, 32(12), 3513-3524.

Gupta, V. K., and Sorooshian, S. (1985). "The automatic calibration of conceptual catchment models using derivative based optimization algorithms." Water Resources Research, 21(4), 473-485.

Huber, W. C., and Dickinson, R. E. (1992). The USEPA SWMM4 Stormwater Management Model, version 4: User's Manual, University of Guelph, Guelph, Ontario. 
Ibrahim, Y., and Liong, S. Y. (1992). "Calibration strategy for urban catchment parameters." ASCE Journal of Hydraulic Engineering, 118(11), 1550-1570.

Ibrahim, Y., and Liong, S. Y. (1993). "A method of estimating optimal catchment model parameters." Water Resources Research, 29(9), 3049-3058.

Javaheri, H. (1999). "Automatic calibration of urban runoff models using global optimization techniques", Master Thesis, Department of Civil Engineering and Applied Mechanics, McGill University, Montreal, Quebec.

Johnston, P. R., and Pilgrim, D. H. (1976). "Parameter optimization for watershed models." Water Resources Research, 12(3), 477-486.

Liong, S. Y., Chan, W. T., and Lum, L. H. (1991). "Knowledge-based system for SWMM runoff component calibration." ASCE Journal of Water Resources Planning and Management, 117(5), 507-523.

Liong, S. Y., and Ibrahim, Y. (1994). "Estimation of peak flow and runoff volume with response surface method." ASCE Journal of Water Resources Planning and Management, 120(2), 161-175.

Liong, S. Y., Chan, W. T., and Shreeram, J. (1995). "Peak-flow forecasting with genetic algorithm and SWMM." ASCE Journal of Hydraulic Engineering, 121(8), 613-617.

Nelder, J. A., and Mead, R. (1965). "A simplex method for functional minimisation." Comput. J., 7(4), 308-313.

Nix, S. J. (1994). Urban Stormwater Modeling and Simulation, CRC Press, Inc.

Viessman, W., and Lewis, G. L. (1996). Introduction to Hydrology, Harper Collins College Publishers.

Wang, Q. J. (1991). "The genetic algorithm and its application to calibrating conceptual rainfall-runoff models." Water resources research, 27(9), 2467-2471.

WP-Software. (1993). XP-SWMM: Stormwater management model with XP graphical Interface. User's manual, version 1.0, WP Software, Belconnen. 